\title{
Immunoprofiling of early, untreated rheumatoid arthritis using mass cytometry reveals an activated basophil subset inversely linked to ACPA status
}

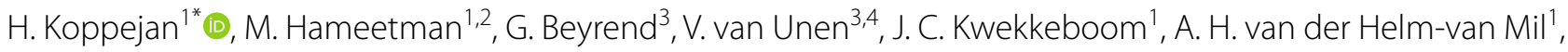
R. E. M. Toes ${ }^{1}$ and F. A. van Gaalen ${ }^{1}$

\begin{abstract}
Background: Autoantibody production is a hallmark of rheumatoid arthritis (RA). Anti-citrullinated protein antibodies (ACPA) are highly disease-specific, and their presence is associated with more severe disease and poor prognosis compared to ACPA-negative patients. However, the immune cell composition associated with antibody-positive/negative disease is incompletely defined. Mass cytometry (MC) is a high-dimensional technique offering new possibilities in the determination of the immune cell composition in rheumatic diseases. Here, we set up a broad phenotyping panel to study the immune cell profile of early untreated RA to investigate if specific immune cell subsets are associated with ACPA+ versus ACPA - RA.

Methods: Freshly obtained PBMCs of early, untreated RA patients (8 ACPA+ and 7 ACPA-) were analysed using a 36-marker $M C$ panel, including markers related to various immune lineages. Data were processed using Cytosplore for dimensional reduction (HSNE) and clustering. Groups were compared using Cytofast. A second validation cohort of cryopreserved PBMCs obtained from early RA patients (27 ACPA+ and 20 ACPA-) was used to confirm MC data by flow cytometry (FC). FC data were processed and analysed using both an unsupervised analysis pipeline and through manual gating.

Results: $M C$ indicated no differences when comparing major immune lineages (i.e. monocytes, $T$ and $B$ cells), but highlighted two innate subsets: $\mathrm{CD}_{2} \mathrm{~L}^{+}$basophils $(p=0.33)$ and a subset of CD16 ${ }^{-}$NK cells $(p=0.063)$. Although the NK cell subset did not replicate by FC, FC replication confirmed the difference in $\mathrm{CD}_{2} \mathrm{~L}^{+}$basophil frequency when comparing ACPA+ to ACPA - patients (mean $0.32 \%$ vs. $0.13 \% ; p=0.01$ ).

Conclusions: Although no differences in major lineages were found between early ACPA + and ACPA - RA, this study identified the reduced presence of activated basophils in ACPA-negative disease as compared to ACPA-positive disease and thereby provides the first evidence for a connection between activated basophils and ACPA status.
\end{abstract}

Keywords: Rheumatoid arthritis, Mass cytometry, Immune-profiling, Basophils, CD62L

*Correspondence: h.j.koppejan@lumc.nl

${ }^{1}$ Department of Rheumatology, Leiden University Medical Center, PO box 9600 (Zone C1-R), Albinusdreef 2, 2233, ZA, Leiden, The Netherlands

Full list of author information is available at the end of the article

\section{Background}

Rheumatoid arthritis (RA), a common chronic autoimmune disease, is characterized by persistent synovial and systemic inflammation, potentially leading to irreversible joint damage. A hallmark of RA is the presence original author(s) and the source, provide a link to the Creative Commons licence, and indicate if changes were made. The images or other third party material in this article are included in the article's Creative Commons licence, unless indicated otherwise in a credit line to the material. If material is not included in the article's Creative Commons licence and your intended use is not permitted by statutory regulation or exceeds the permitted use, you will need to obtain permission directly from the copyright holder. To view a copy of this licence, visit http://creativecommons.org/licenses/by/4.0/. The Creative Commons Public Domain Dedication waiver (http://creativeco mmons.org/publicdomain/zero/1.0/) applies to the data made available in this article, unless otherwise stated in a credit line to the data. 
of autoantibodies, such as rheumatoid factor (RF) and anti-citrullinated protein antibodies (ACPA). ACPA are highly disease-specific, and their presence is associated with more severe disease and poor prognosis compared to ACPA-negative patients (ACPA-) [1-3].

The pathogenesis of RA is incompletely understood, apart from a role for B cells, given the efficacy of B celldepleting therapies $[4,5]$. Despite great efforts by the field, data on the immune cell composition truly associated with RA is limited. Several studies investigating RA on the immune cell level report on decreased frequencies of regulatory $\mathrm{T}$ cells in RA patients [6]. Likewise, CCR6 expression was reported to be upregulated in $\mathrm{CD}_{4}{ }^{+} \mathrm{CD} 45 \mathrm{RO}^{+} \mathrm{T}$ helper cells in ACPA-positive $(\mathrm{ACPA}+)$ disease, as was an increase in the frequency of CD4, CD8 double-positive T cells and the ratio of M1/ M2 monocytes in peripheral blood [7-9]. In contrast, a signature $\mathrm{CD} 4^{+} \mathrm{T}$ cells compatible with IL-6-mediated STAT3 signalling was observed mostly in ACPA - disease during the early clinical phase [10]. These studies generally focused on a specific cell type or immune cell subset present in the immune system providing limited information on the overall cellular composition of the major immune lineages in RA and/or RA endotypes. Moreover, few findings have been replicated which could be due to treatment effects or differences in disease duration.

The analyses of the immune cell composition by highdimensional single-cell platforms such as mass cytometry $(\mathrm{MC})$, also known as cytometry by time of flight, offer new possibilities to gain additional insights into the cellular composition within rheumatic diseases. $\mathrm{MC}$ is an antibody-based technique utilizing heavy metal isotopeconjugated probes [11]. Until now, MC has been used only to a limited extent to investigate rheumatic diseases. Nonetheless, the first studies using MC have revealed novel insights as suggesting, for example, that that PD- $1{ }^{\text {hi }}$ CXCR $5^{-} \mathrm{CD} 4^{+} \mathrm{T}$ cells (Tph cells) are expanded in joints and blood of seropositive RA [12]. Other studies using MC specifically reported differences in $\mathrm{T}$ and $B$ cell, monocytes and neutrophils in RA as compared to controls but not comparing ACPA-positive and ACPAnegative disease $[5,13-15]$.

Given that in RA the production of ACPA antibodies is associated with a more severe clinical course, we hypothesized that differences at the immune cell level and in particular in $\mathrm{T}$ and $\mathrm{B}$ cell compartments are detectable between ACPA+ and ACPA- RA. Since MC allows the detailed profiling of the immune cell composition in RA, we now sought to probe the immune system in early, untreated RA, reducing possible confounding factors associated with treatment or symptom duration. Here, we describe an immune profile of early untreated RA to investigate if specific immune cells or subsets could be associated with the differences observed on a clinical level.

\section{Methods \\ Patient material}

For mass cytometry (MC) experiments, heparin blood was collected from untreated patients with polyarthritis of recent onset, who were enrolled into the Leiden Early Arthritis Clinic (EAC) cohort study (baseline EAC visit) [16]. All patients were diagnosed with RA and fulfilled the 2010 classification criteria for RA within the first year of follow-up (ACPA $+n=8, \mathrm{ACPA}-n=7)$.

For flow cytometry (FC) experiments, peripheral blood mononuclear cells (PBMCs) were previously collected from RA patients enrolled in the EAC study and cryopreserved until use. A total of 47 patients diagnosed with RA (27 ACPA+ and 20 ACPA-) fulfilling the 1987 ACR criteria [17] were randomly selected. The EAC study was approved by the LUMC ethical committee, and all patients provided written informed consent. The patient's characteristics are summarized in Table 1.

\section{Sample processing for mass cytometry}

PBMC isolation from heparin blood was performed by Ficoll-Paque gradient centrifugation. Freshly isolated PBMCs $\left(3 \times 10^{6}\right.$ cells $)$ were directly stained with CellID intercalator-103Rh to identify dead cells (Fluidigm, South San Francisco, CA, USA). Upon ${ }^{103}$ Rho staining, cells were fixed in freshly prepared $1.85 \%$ formaldehyde solution (Sigma-Aldrich, Darmstadt, Germany; diluted in Maxpar PBS, Fluidigm) and stored in Maxpar Cell staining buffer (Fluidigm) at 4C. Fixed samples were stored in suspension for a maximum of 3 days.

\section{Staining and acquisition for mass cytometry}

All MC antibodies and their respective suppliers are summarized in Supplementary Table 1. Antibodies were either pre-conjugated to metals (Fluidigm) or inhouse conjugated at $100-\mu \mathrm{g}$ scale using a Maxpar X8 antibody labelling kit according to the manufacturer's protocol (Fluidigm). All in-house conjugated antibodies were stored in $100 \mu \mathrm{l}$ antibody stabilization buffer supplemented with $0.05 \%$ sodium azide. All antibodies (pre- or in-house conjugated) were tested in a serial dilution staining to determine the optimal signal with minimal background. Staining was performed according to the manufacturer's protocol (Fluidigm). Briefly, freshly fixed PBMCs were pre-incubated with Fc-block (Human TruStain FcX, Biolegend, San Diego, CA, USA), stained for 35 surface markers in the presence of Fc-block, permeabilized (eBioscience Foxp3/transcription factor staining buffer set, eBioscience, San Diego, CA, USA), and stained for intranuclear Ki-67, and finally, all cells are 
Table 1 Patient clinical characteristics of rheumatoid arthritis patients used in this study

\begin{tabular}{|c|c|c|c|c|}
\hline & $\begin{array}{l}\text { MC } \\
\mathrm{RA} \mathrm{ACPA}^{+} \\
n=8\end{array}$ & $\begin{array}{l}\text { MC } \\
\text { RA ACPA }^{-} \\
n=7\end{array}$ & $\begin{array}{l}\mathrm{FC} \\
\mathrm{RA} \mathrm{ACPA}^{+} \\
n=27\end{array}$ & $\begin{array}{l}\mathrm{FC} \\
\mathrm{RA} \mathrm{ACPA}^{-} \\
n=20\end{array}$ \\
\hline Age in years, average (range) & $64(48-75)$ & $67(54-79)$ & $55(17-81)$ & $51(21-86)$ \\
\hline Females, $n(\%)$ & $4(50)$ & $4(57)$ & $16(59)$ & $12(60)$ \\
\hline RF positive, $n(\%)$ & $8(100)$ & $2(29)$ & 19(79) & $5(21)$ \\
\hline Elevated CRP, $n(\%)$ & $5(50)^{\mathrm{a}}$ & $3(25)$ & $62(24)$ & $38(15)$ \\
\hline SJC, mean (range) ${ }^{b}$ & $9(3-20)$ & $14(4-29)$ & $5(0-20)$ & $3(0-16)$ \\
\hline TJC, mean (range) ${ }^{b}$ & $11(2-20)$ & $17(1-34)$ & $3(0-16)$ & $3(0-18)$ \\
\hline DMARD usage, $n(\%)^{c}$ & $0(0)$ & $0(0)$ & $6(31 \%)$ & $4(36 \%)$ \\
\hline Disease duration (in days), average (range) ${ }^{d}$ & $132(13-600)$ & $42(21-60)$ & $108(5-542)$ & $132(3-610)$ \\
\hline
\end{tabular}

${ }^{a}$ No CRP was recorded for one male and one female (male $E S R=34$, female $E S R=126$ )

${ }^{\mathrm{b}}$ For $3 \mathrm{ACPA}+$ and 2 ACPA-, no SJC/TJC was recorded in the FC dataset

${ }^{\mathrm{C}}$ For 17 RA patients, no DMARD usage was recorded in the FC dataset, frequency of DMARD calculation based on $n=19$ in ACPA + and $n=11$ in ACPA-. DMARD usage included hydroxychloroquine, sulfasalazine and methotrexate

${ }^{\mathrm{d}}$ For $1 \mathrm{ACPA}+$ and $1 \mathrm{ACPA}-$, no disease duration was recorded in the FC dataset

$M C$ mass cytometry, FC flow cytometry, ACPA anti-citrullinated protein antibodies, RF rheumatoid factor, CRPC-reactive protein, SJC swollen joint count, TJC tender joint count, $D M A R D$ disease-modifying anti-rheumatic drugs

stained with Cell-ID intercalator-Iridium $\left({ }^{191} \mathrm{Ir}\right.$ and ${ }^{193} \mathrm{Ir}$, Fluidigm) diluted into Maxpar Fix and Perm Buffer (Fluidigm). Samples were stored as a pellet at $4{ }^{\circ} \mathrm{C}$ for a maximum of 3 days until $\mathrm{MC}$ acquisition. Prior to acquisition, samples were washed with Maxpar Cell Staining buffer and Maxpar Water (Fluidigm). Cells were diluted in Maxpar Water (Fluidigm) to approx. $0.75 \times 10^{6} \mathrm{cells} / \mathrm{ml}$ and $10 \% \mathrm{v} / \mathrm{v}$ EQ beads were added (EQ Four Element Calibration Beads, Fluidigm). Samples were acquired on a Fluidigm Helios CyTOF system using HT injector, normalized using reference EQ passport $\mathrm{P} 13 \mathrm{H} 2302$ within the Fluidigm acquisition software, and converted to FCS files. To monitor technical variation, a cryopreserved PBMC reference sample was included (buffy coat obtained from Sanquin, The Netherlands).

\section{Mass cytometry data analysis}

FCS files were manually processed in Flowjo v10 (TreeStar, Ashland, OR, USA) to gate single, live, $\mathrm{CD} 45^{+}$cells and exported into new FCS files (Supplementary Figure 1). All processed samples combined resulted in a dataset of $6.4 \times 10^{6} \mathrm{CD} 45^{+}$cells originating from 15 different samples.

All $\mathrm{CD} 45^{+}$cells were sample-tagged, hyperbolic ArcSinh-5 transformed, and simultaneously subjected to dimension reduction in Cytosplore (v2.2) [18]. We used hierarchical stochastic neighbour embedding (HSNE) [19], preventing data loss linked to downsampling prior to dimension reduction and clustering methods. Major immune lineages were identified through the use of a 5-level HSNE taking all 36 markers into account, using default perplexity and iterations (30 and 1000, respectively). Individual lineages were further investigated within Cytosplore by 'zoom in' to a maximum of $0.3 \times 10^{6}$ landmarks. Both overview and 'zoomed' levels were subjected to Gaussian mean shift (GMS) clustering within Cytosplore. Clusters were exported as new FCS files, including sample tags. Downstream analysis and comparisons were performed using Cytofast [20]. The generated marker expression heatmap and HSNE map acted as guidelines to determine the expression pattern for clusters of interest. The selected markers were used in a flow cytometry panel to perform an independent replication study on a second set of EAC samples as confirmation of the MC outcome.

\section{Flow cytometry}

In a separate experiment, two cell subsets identified by $\mathrm{MC}$ were investigated using flow cytometry (FC). To this end, PBMCs of 47 untreated early RA patients (not previously used in MC) were thawed and stained with Zombie Yellow fixable Viability Kit (Biolegend). Samples were stained for surface markers resembling clusters of interest identified with $\mathrm{MC}$, using BV605 as a general dump channel to exclude non-relevant cells. FC samples were used directly without the need of fixation. All FC antibodies and their respective suppliers are listed in Supplementary Table 2. As each antibody clone may have a slightly different epitope, we ensured that clones used in FC are similar to those used in MC. All samples were acquired and unmixed on a 3-laser Cytek ${ }^{\mathrm{TM}}$ Aurora spectral cytometer (Cytek Biosciences Inc, Fremont, CA, USA). In contrast to conventional flow, spectral flow cytometry captures the full emission spectrum of every 
fluorescent molecule and allows unmixing based on the differences in the overall spectral signatures. FC samples were processed using FlowJo v10 for gating of single/live/ $\mathrm{CD} 45^{+}$cells (Supplementary Figure 2). Pre-processed samples were either analysed using Cytosplore or manual gating in FlowJo. The ArcSinh cofactor needed for Cytosplore analysis was determined for each fluorochrome separately and applied to all samples as described by Melsen et al. (Github: https://github.com/janinemelsen/ Single-cell-analysis-flow-cytometry) [21]. In case of manual analysis, pre-gated $\mathrm{CD} 45^{+}$samples were further gated in FlowJo v10 based on the expression pattern observed in the MC data.

Statistical analysis was performed in $\mathrm{R}$ using Cytofast when analysing $>2$ clusters simultaneously (MC data only) or using GraphPad Prism v8 (GraphPad Software, La Jolla, CA, USA). Statistical significance was considered when $p<0.05$.

\section{Results}

Analyses of peripheral blood cells from early ACPA+ and ACPA- RA patients by a 36-marker MC panel

After applying the 36-marker mass cytometry (MC) panel to 15 untreated, early ACPA+ and ACPA- RA patients (Table 1), we first performed a 5-level HSNE dimension reduction on all 15 samples simultaneously, showing a clear separation of different major immune lineages such as T cells, B cells and monocytes (Fig. 1A, B, dashed lines). The HSNE [17] map also depicted landmarks representing the innate lineage (Fig. 1A, solid line). Default clustering in Cytosplore [22] resulted in 21 different clusters at the overview level (Fig. 1C). Analyses of these clusters by Cytofast [18] indicated that none of the cluster frequencies was significantly different between ACPA+ and ACPA- RA (Supplementary Figure 3). Although some of the ACPA - samples clustered together, this was not consistent for all ACPA-negative patients (Supplementary Figure $3 \mathrm{~A}$ ). Nonetheless, a trend towards the increased presence of $\mathrm{CD} 27^{\text {low }} \mathrm{CD} 28^{\text {low }} \mathrm{CD} 45 \mathrm{RA}^{+} \mathrm{CD} 4^{+}$ $\mathrm{T}$ cells (cluster 21) was observed in ACPA+ patients (3/8 patients), as this cluster was absent in all ACPA - patients (Supplementary Figure 3C). This cluster probably represents a subset of terminally differentiated effector memory $\mathrm{T}$ cells $\left(\mathrm{T}_{\mathrm{EMRA}}\right)$ based on their loss of $\mathrm{CD} 27 / \mathrm{CD} 28$ combined with the expression of CD45RA.

In agreement with the results presented above, manual gating of the major immune lineages in FlowJo did not result in any significant differences (Fig. 1D), confirming that the overall composition of the major immune cell lineages in peripheral blood is similar between ACPA - and ACPA+ patients.

\section{HSNE of solely innate lineage identified two clusters: differences in basophil and NK cell subsets}

Next, we investigated whether differences would become apparent when analysing subsets on a more detailed level. To this end, Cytosplore was used to zoom in on each lineage, analysing CD4 T cells, CD8 T cells, monocytes, B cells and the innate subsets in a separate analysis (zooming in to a maximum of $0.3 \times 10^{6}$ landmarks in a single HSNE map). We did not identify any (significant) differences when analysing the major lineage subsets separately (data not shown).

All the innate subsets combined represented $14.5 \%$ of the total data set $\left(0.93 \times 10^{6}\right.$ out of $6.4 \times 10^{6}$ cells $)$, and default clustering resulted in 30 clusters (Fig. 2A). To delineate the cluster frequency distribution for ACPA+ and ACPA - samples, we used Cytofast revealing that 2 clusters were differentially present in ACPA+ vs. ACPA - patients: clusters 18 and 24 (Fig. 2A-black boxes). The frequency of cluster 18 was significantly lower in ACPA- RA $(p=0.033)$, whereas the frequency of cluster 24 appeared higher in ACPA- RA ( $p$ $=0.063$ ) (Fig. 2B). Furthermore, applying a dimension reduction based on cell cluster 18 and 24 frequencies indicated a clear separation of samples when colourcoded for ACPA status (Fig. 2C).

Cluster 18 expressed markers linked to basophils such as FceRI, CD123, CD45RO, and additionally expressed CD25, CD62L and CCR6 (Fig. 2A, D). The remaining FceRI-negative clusters appeared to be more similar in $\mathrm{ACPA}-$ and ACPA+ RA, though differences in expression can be observed, e.g. for CD8 or CCR6. Cluster 24 was lineage-negative (incl. HLA-DR and CD16) and expressed markers often observed on $\mathrm{CD} 16^{-} \mathrm{NK}$ cells or innate lymphocyte cells (ILCs) such as CD56, CD94 and CD161 (Fig. 2A, D). As no clear CD127 staining is observed, we indicate cluster 24 as a subset of NK cells.

\section{Flow cytometry replication-confirmation of $\mathrm{CD} 62 \mathrm{~L}^{+}$ basophils inversy linked to ACPA status}

To confirm the MC findings, we next performed a replication study using flow cytometry (FC) and an

(See figure on next page.)

Fig. 1 Analysis of major lineages revealed no differences between ACPA+ and ACPA- patients. A Five-level HSNE separated all major lineages as indicated by dashed circles, colour-coded for ACPA status (positive $=$ red, negative $=$ blue). The solid circle indicates innate landmarks. $\mathbf{B}$ Examples of expression patterns of the HSNE map shown in A. C Heatmap of clusters identified through default clustering in Cytosplore (Gaussian mean shift) showing the expression pattern. D Manual gating of major lineages showed no significant difference in frequency between ACPA+ and ACPA- 

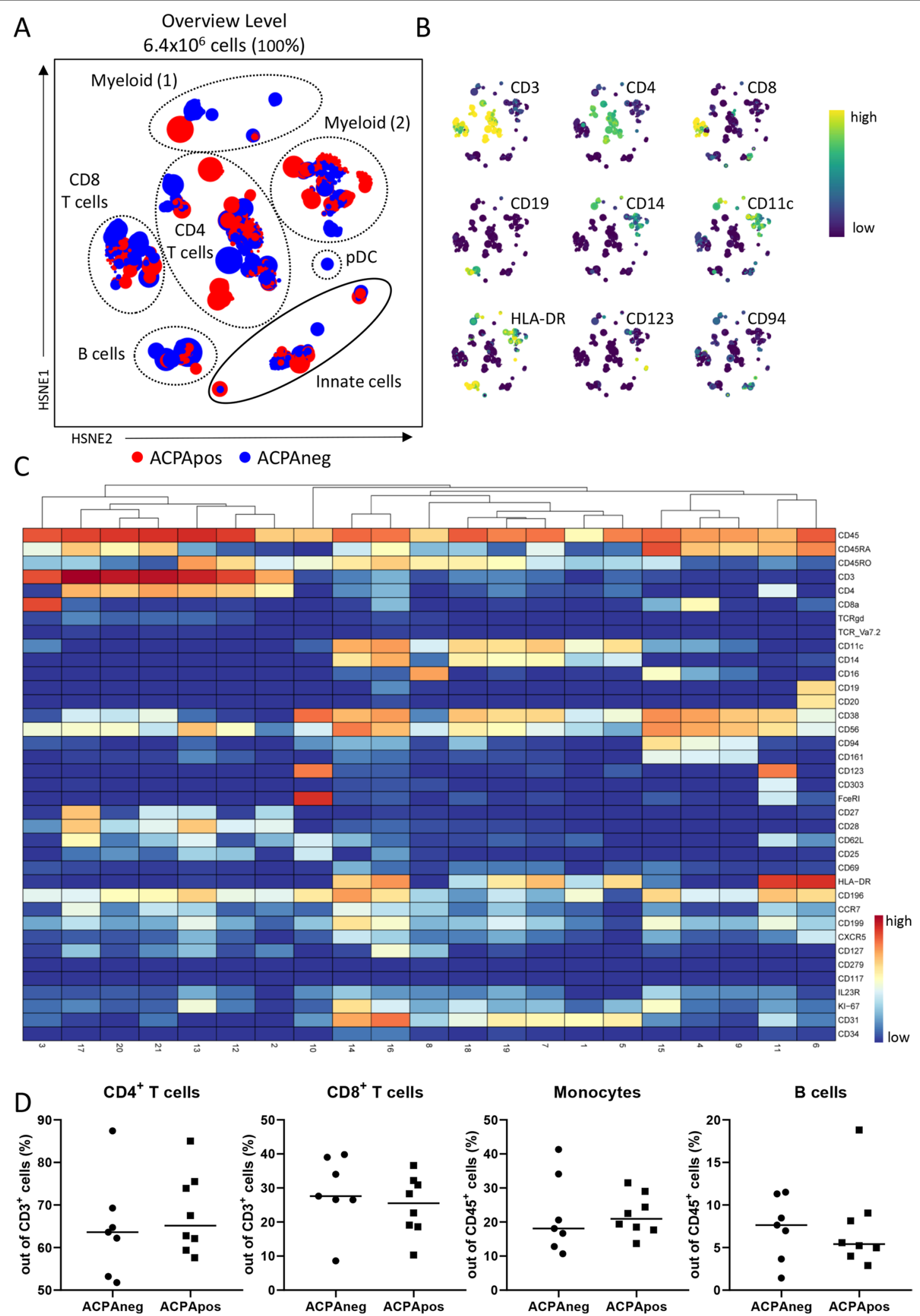

Fig. 1 (See legend on previous page.) 
independent cohort of cryopreserved PBMCs obtained from early RA patients $(n=47 ; 27$ ACPA+ vs. 20 ACPA-). All samples were split and part was used for confirmation of the basophil subset. Likewise, although the differences in NK cell subset distribution showed a clear trend but had not reached statistical significance in the discovery MC study, also the NK cell subset distribution was analysed by FC. The FC panels were designed based on the marker expression, as shown in Fig. 2A, related to either cluster 18 (panel 1) or cluster 24 (panel 2). The antibodies for FC experiments used are listed in Supplementary Table 2. FC data were pregated for single, live, $\mathrm{CD} 45^{+}$cells and exported into new FCS files, similar to MC data processing (Supplementary Figure 2). Processed files were analysed both by unsupervised analysis and manual gating. Unsupervised dimension reduction of panel 1 samples indicated a small dump-negative subset that separated from the rest of the cells (Fig. 3A). The expression pattern of this subset matched that of MC cluster 18: $\mathrm{CD} 123{ }^{\mathrm{hi}} \mathrm{FcERI}^{+} \mathrm{CD} 45 \mathrm{RO}^{\text {med }}$. Intriguingly, this subset included both ACPA+ and ACPA- cells; however, only the ACPA+ cells expressed CD62L (Fig. 3A, top left and bottom right panel). These data validate our first MC findings by identifying differences between ACPA+ and ACPA - samples in the presence of a $\mathrm{CD}_{2} \mathrm{~L}^{+}$basophil-like cell population.

Based on the unsupervised FC pipeline, we determined a manual gating strategy in Flowjo for cluster 18 within the pre-gated $\mathrm{CD} 45^{+}$cells. To this end, four separate gates were drawn: dump negative, CD45RO positive, CD123 ${ }^{\text {hi }}$ FceRI ${ }^{\text {hi }}$ and CD62L positive. Boolean gating was applied to all four gates simultaneously, resulting in a subset of cells solely including cells positive for all four gates, mimicking the Cytosplore dimension reduction (Fig. 3B). Comparing ACPA+ and ACPA - patients measured by FC confirmed the MC findings as the frequency of this FC cluster was lower in ACPA-RA (mean $0.32 \%$ vs. $0.13 \%$; $p=0.01$, Fig. $3 C$ ). Comparing the frequency of manually gated cluster 18 to a small group of 6 healthy controls (HC) showed that $\mathrm{HC}$ (mean $0.32 \%$ ) were more similar to ACPA+ patients and not ACPA-.

Dimension reduction of panel 2 samples resulted in a large dump-negative islet (Supplementary Figure 4). A small section of this islet partially mimicked MC cluster
24 (dashed circle), indicated by lack of CD16 and CD8 expression combined with the presence of CD45RA expression. The remainder of the markers related to cluster 24 could not clearly be identified within this section, nor was there a clear separation of cells originating from either ACPA + or ACPA- samples. The presence of the NK subset in these cryopreserved samples therefore remains inconclusive.

\section{Discussion}

RA patients are often separated based on their ACPA status, as the prognosis of ACPA+ is worse compared to ACPA-. Currently, a more comprehensive immune cell profile linked to ACPA status is lacking. During this study, we investigated the PBMC immune cell profile of ACPA+ and ACPA- RA at an early stage of the disease, prior to treatment initiation, using a 36-marker $\mathrm{MC}$ panel. Upon investigating major immune lineages, no differences were observed between ACPA+ and ACPApatients. However, comparing early untreated ACPA+ and ACPA- RA by MC did reveal differences in two specific clusters within the innate populations. The first cluster represented a basophil-like subset expressing CD62L, CD25 and CCR6, present mostly in ACPA+ patients. The second cluster represented a $\mathrm{CD} 16^{-} \mathrm{NK}$ cell subset present mostly in ACPA- patients. Flow cytometry (FC) replication was performed using an independent cohort of RA patients. FC experiments confirmed the basophil $\mathrm{MC}$ results, showing $\mathrm{CD} 62 \mathrm{~L}^{+}$basophils are reduced in ACPA-patients, as identified both by unsupervised analysis and manual gating. Moreover, manual gating of healthy control (HC) samples indicated that $\mathrm{Cl}-18$ is indeed reduced in APCA-, as $\mathrm{HC}$ were more similar to $\mathrm{ACPA}+$.

The NK subset identified by MC could not be confirmed by FC replication. The reason for this lack of replication is unknown. Still, it could relate to the relatively low number of patients included in the discovery cohort, the use of cells that had undergone a freeze/thaw cycle, the difference between fixed (MC) or unfixed (FC) samples, and/or differences with the patient population used for the replication studies with FC as these patients were not all naïve to therapy.

Based on previously published literature on the role of e.g. B cells and $T$ cells in RA, we expected to find more apparent differences between these subsets when

(See figure on next page.)

Fig. 2 HSNE analysis of all innate subsets indicated differences in basophil and NK cell subsets. A Heatmap representing all clusters within the innate population and their respective expression patterns, cluster 18/24 indicated with black boxes. B Cluster 18 (basophil subset, $p=0.033$ ) was reduced in ACPA - patients and there was a strong trend for cluster 24 (NK cell subset $p=0.063$ ) present only in ACPA- patients (Cytofast). C tSNE plotting samples using clusters 18 and 24 as input confirmed the separation shown in B. D HSNE map indicating cluster 18 and cluster 24 (dashed circles) and examples of the expressed markers. ACPA+ in red, ACPA - in blue 

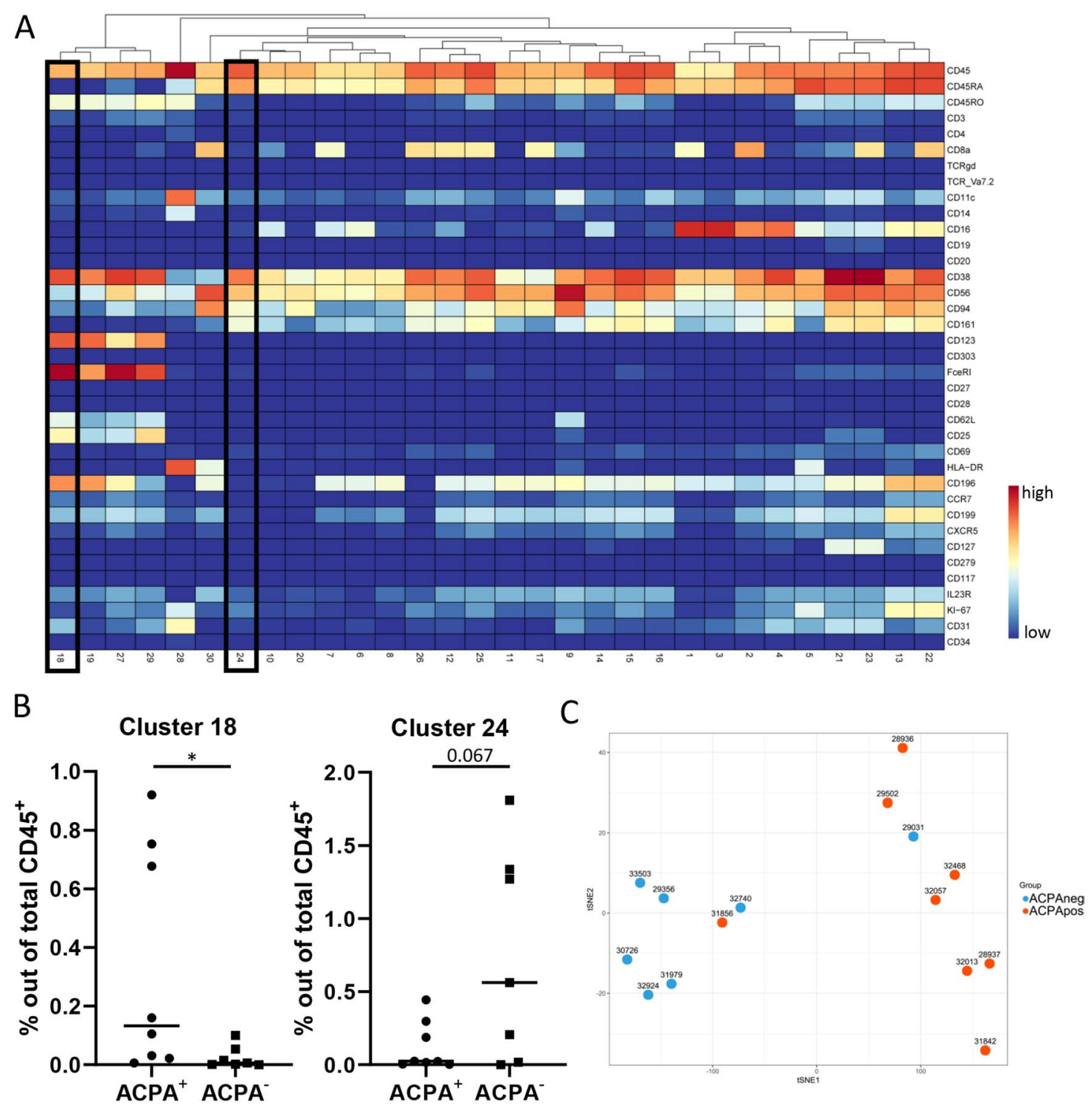

D
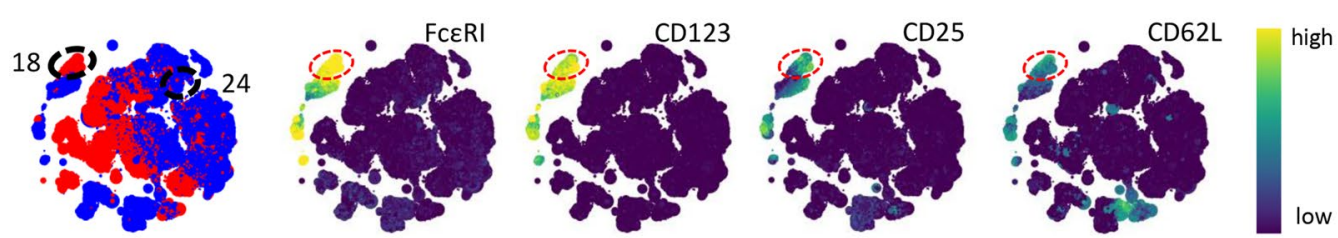

- ACPApos - ACPAneg
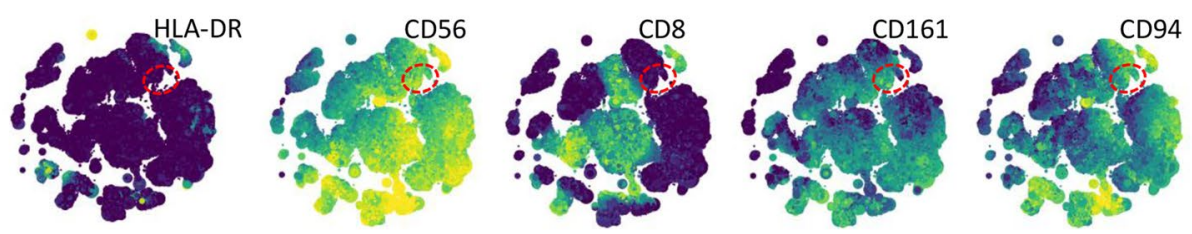

Fig. 2 (See legend on previous page.) 

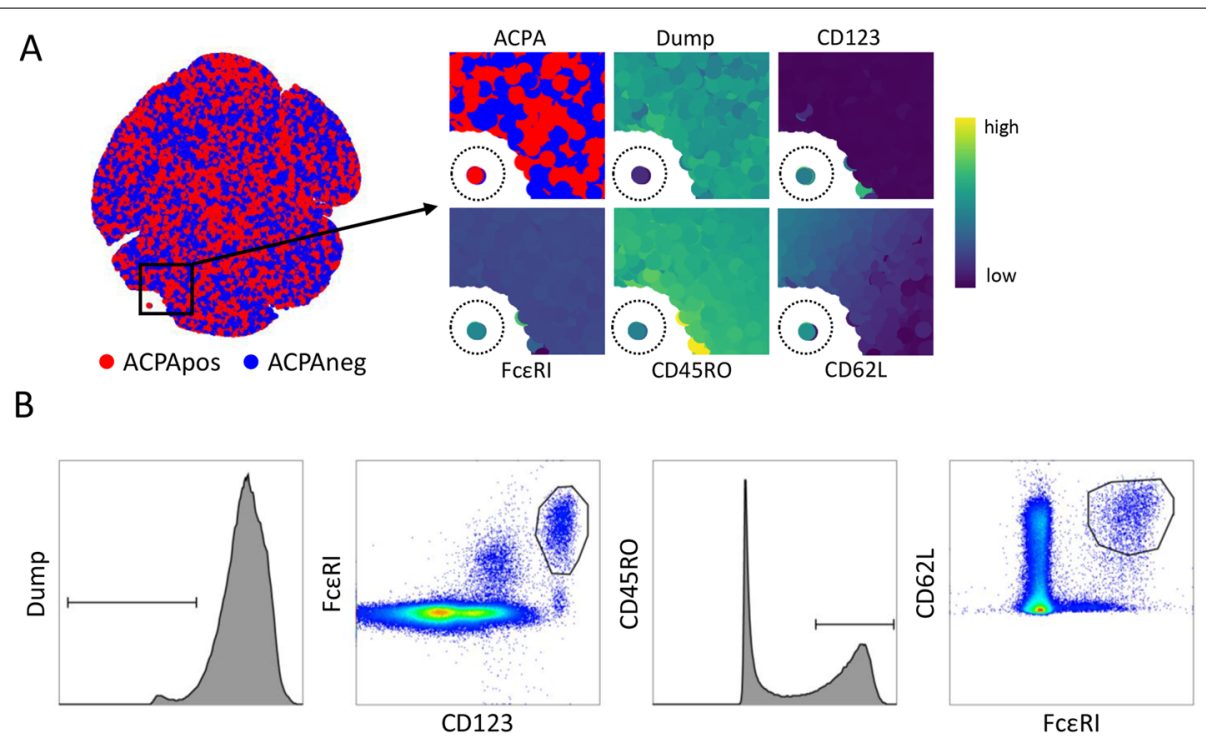

C

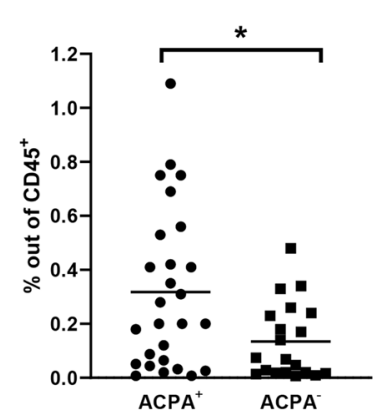

Fig. 3 Independent flow cytometry replication confirmed the reduced presence of CD62 $\mathrm{L}^{+}$basophils in ACPA- samples. A Cytosplore dimension reduction of manually transformed flow FCS files of panel 1 showing both ACPA+ (red) and ACPA- (blue). Smaller panels on the right represent the cells within the backbox. Expression patterns matched that of MC cluster 18 (dashed circle), including CD123, FceRl and CD45RO. CD62L expression was linked to ACPA status. B Gates used in Boolean gating in Flowjo to manually gate cluster 18. C Frequency of manually gated cells representing MC cluster 18 . Subset frequency was lower in ACPA-RA (mean $0.32 \%$ vs $0.13 \% ; p=0.01$ )

comparing major lineages. However, our MC study did not show any differences in major immune lineages. Possible explanations for this outcome could be related to the fact that a few of the MC ACPA- patients are RF+ and therefore were not completely autoantibody negative. The study size did not allow further stratification on other factors such as RF. Additionally, we included a relatively small number of patients in the 'discovery study' by $\mathrm{MC}$ analyses, and consequently, we might be underpowered to identify differences that have been reported previously. Lastly, our panel consisted of various markers to be able to investigate the overall immune cell profile of RA patients instead of running dedicated, detailed panels studying either $\mathrm{T}$ or B cells specifically. This could also contribute to the apparent similar distribution of $\mathrm{T}$ and $\mathrm{B}$ cell subsets in peripheral blood of ACPA - and ACPA+ patients.
Nonetheless, this study showed the value of high parameter analysis to allow broad immune cell screening of inflammatory rheumatic diseases. High-dimensional analysis reduces bias as more markers can be combined in a single experiment, resulting in the identification of unexpected cell subsets. Furthermore, our study also clearly highlighted the importance of replication: the NK cell subset observed in ACPA- disease identified by MC could not be identified by FC, indicating it is not clearly linked to disease status. This emphasizes the need for independent replication cohorts in MC studies. In contrast to the NK cell subset, the MC results for the basophil-like subset were successfully replicated by FC. The FC study included more patients than our initial $\mathrm{MC}$ discovery study, and therefore, these results are more accurate. 
Our study utilizes PBMCs instead of whole blood, which may bias our outcome since one of the identified subsets belongs to the granulocyte lineage. Although a Ficoll-Paque gradient isolation of basophils is not uncommon, we cannot rule out that whole blood assessment of this specific CD62L+ basophil subset would have yielded different results. However, we did measure a small subset of healthy paired WB/PBMC samples and were able to identify CD62L+ basophils to a similar extend. Using PBMCs as readout may not reflect what happens in situ in the inflamed joint, but unfortunately, we were not able to obtain synovial fluid from untreated patients to further investigate this. However, a great strength of our study is the inclusion of DMARD-naïve patients in the MC study, allowing a more accurate measurement of the patient's immune cell profile without treatment bias. Likewise, our MC study used freshly isolated PBMC samples. Certain cell types are sensitive to cryopreservation, such as e.g. plasma cells. By analysing fresh samples, there will be no (selective) cell loss due to a freeze/thaw cycle and the immune cell profile obtained is better reflecting the in situ PBMC immune cell profile. As most studies often included cryopreserved samples, this may be another reason why our study was not able to replicate reported differences between ACPA+ and ACPA- RA patients.

Cells comprising cluster 18, reduced in ACPA- samples, showed similarities with basophils based on CD123/ FcERI/CD45RO expression, the lack of (plasmacytoid) DC markers HLA-DR/CD45RA/CD303 and absence of major lineage markers. Ficoll-Paque gradient centrifugation was performed to remove erythrocytes and granulocytes from the PBMCs; however, basophils can remain within the monolayer $[20,21]$. Cytosplore analysis of MC samples indicated that cluster 18 could be separated from other $\mathrm{CD} 123^{+} / \mathrm{FcERI}^{\mathrm{hi}}$ subsets based on the expression of CD25, CD62L and CCR6. An independent FC replication supported the presence of 'cluster 18 basophil-like cells' identified by $\mathrm{MC}$, both by dimension reduction and manual gating. Of note, the difference between ACPA+ and ACPA - was predominantly driven by CD62L expression.

The reason why $\mathrm{CD} 62 \mathrm{~L}+$ basophil numbers are reduced in the blood of ACPA - patients as compared to ACPA+ subjects is unknown. CD62L, also known as L-selectin, is an adhesion molecule associated with early activation and rolling of leukocytes along the vessel wall. Adhesion molecules are often affected by cryopreservation resulting in decreased intensity as has been described for CD62L on T cells and CD34+ ${ }^{+}$cells specifically [23-27]. This further emphasizes the need for the use of freshly collected material and could be a possible explanation as to why our findings have not been reported previously. Of interest, our FC cohort still indicated a difference in CD62L expression in basophils, despite the fact the samples were cryopreserved. As the expression of CD62L is very high, a decrease may not be crucial when investigating the basophils specifically in contrast to other subsets such as T cells. CD62L has been reported to be expressed by activated basophils, pointing to a possible role of these cells in the disease process $[28,29]$. Although speculation, basophils could contribute to inflammatory processes in several ways. For example, data obtained in preclinical mouse models suggest a role of activated basophils in immunological memory response as depletion of basophils lowered the humoral memory response on both the $\mathrm{T}$ cell and $\mathrm{B}$ cell level, a phenomenon possibly related to the ability of basophils to bind intact antigen on their cell surface [30]. Likewise, co-culture of activated basophils with $\mathrm{T}$ and B cells supported B cell function and induced a 'B helper' phenotype in $\mathrm{CD}^{+}{ }^{+} \mathrm{T}$ cells. In human studies, $\mathrm{CD} 2 \mathrm{~L}^{+}$basophils have been linked to kidney diseases such as chronic kidney disease and lupus nephritis, but their contribution to disease is ill-defined [28, 29]. Considering that activated basophils could support humoral responses, it is intriguing to note that a basophil subset with an activated phenotype is less common in our ACPA - samples. It would be interesting to continue in situ studies to learn more on this subset and see if lower frequencies in the periphery correlate to increased frequencies in inflamed joints. Clearly, additional studies should be performed to investigate activated basophils in the context of autoimmunity. Moreover, it would be very interesting to investigate if the activated basophils in ACPA+ disease are actually linked to the polarization of PD- $1^{\text {hi }} \mathrm{CXCR} 5{ }^{-} \mathrm{CD} 4^{+} \mathrm{Tph}$ cells described previously [12].

\section{Conclusions}

Our data show a reduced population of innate cells with an activated basophil-like phenotype in ACPA- RA. The possible role of these cells in the immune response associated with RA or in other (auto)immune responses is presently unclear. However, our data provide the first evidence of a reduction of activated basophil-like cells in ACPA- disease as well as a rationale to determine their possible contribution to disease.

\section{Abbreviations}

ACPA: Anti-citrullinated protein antibodies; ACR: American College of Rheumatology; DMARD: Disease-modifying anti-rheumatic drugs; EAC: Early Arthritis Cohort; FC: Flow cytometry; FCS: Flow cytometry standard; GMS: Gaussian mean shift; HSNE: Hierarchical stochastic neighbour embedding; ILC: Innate lymphocyte cell; MC: Mass cytometry; MFI: Mean fluorescence intensity; NK 
cell: Natural killer cell; PBMC: Peripheral blood mononuclear cell; RA: Rheumatoid arthritis; RF: Rheumatoid factor; tSNE: t-distributed stochastic neighbour embedding.

\section{Supplementary Information}

The online version contains supplementary material available at https://doi. org/10.1186/s13075-021-02630-8.

Additional file 1: Supplementary Table 1: Antibodies used for Mass

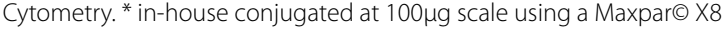
antibody labeling kit (Fluidigm)

Additional file 2: Supplementary Table 2: Antibodies used for Flow Cytometry. Antibodies used in FC experiments to investigate frequencies Cluster 18 (panel 1) and Cluster 24 (panel 2) identified by MC.

Additional file 3: Supplementary Figure 1: Gating strategy for data cleanup of Mass Cytometry data files. MC FCS files are normalized using EQ passport P13H2302 within the Fluidigm software. Gating of normalized files included sequential gating of DNA2- ${ }^{193}$ Irridium vs Event length, followed by DNA1- ${ }^{191}$ Irridium vs DNA2- ${ }^{193}$ Irridium to remove doublets and debris. Cells negative for ${ }^{103}$ Rhodium are selected as live cells. ${ }^{140}$ Cerium negative cells are selected to remove EQ beads $\left({ }^{140} \mathrm{Ce}+\right)$ followed by a selection for $\mathrm{CD}_{4} 5^{+}$cells. Only single/live/bead-free/CD45 ${ }^{+}$cells are exported into a new FCS file which was used for downstream analysis.

Additional file 4: Supplementary Figure 2: Gating strategy for data cleanup of Flow Cytometry files. Flow Cytometry files were unmixed within the CYTEK acquisition software based on single stained controls. Forward and Side-scatter Area are used to remove most debris. Single cells are defined through combination of Height and Area, both for Forward and Side-scatter. Live cells are defined as ZOMBIE-yellow negative. All samples are pre-gated for $\mathrm{CD}^{+} 5^{+}$and exported as new FCS file, similar to MC data processing.

Additional file 5: Supplementary Figure 3: Cytofast analysis of overview level HSNE of Mass Cytometry data. Cytosplore was used to perform dimension reduction and clustering, clusters were exported as new FCS files and loaded in R to be analyzed using the Cytofast package. A) Sample tSNE representing all MC samples. No clear pattern or separation by group was observed. B) Heatmap representing the frequency of each cluster within an MC EAC sample. The order of rows is based on the similarity of cluster-distribution within a given sample compared to other samples. C) Cytosplore analysis of all 21 clusters showing dotplots per cluster and the corresponding frequency per sample separated for ACPA. The overall distribution appears similar; however, a trend is observed for cluster 21 $\left(\mathrm{T}_{\text {EMRA }}\right)$ present in in a few $\mathrm{ACPA}^{+}$only. (ACPA+=red ACPA- =blue)

Additional file 6: Supplementary Figure 4: Unsupervised analysis flow replication of cluster 24 remains inconclusive. Cytosplore dimension reduction of manually transformed flow FCS files of panel 2 showing both ACPA+ (red) and ACPA- (blue). Smaller panels on the right show examples of expression per marker. Red dashed circle indicate cells thar

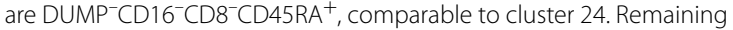
markers linked to cluster 24 (CD56, CD94, CD38 and CD161) did not clearly overlap nor did they separate based on ACPA status. Identification of cluster 24 could not be repeated by FC.

\section{Acknowledgements}

The authors would like to thank Cristina Teodosio, Ellis Niemantsverdriet and El-ad David Amir.

\section{Authors' contributions}

FAvG, HK, MH, REMT and VvU designed the study and experiments. AHvdHvM supplied patient samples. HK, JCK and MH conducted the experiments and acquired all data. FvG, GB, HK, MH and REMT analysed the data. FvG and HK drafted the manuscript. All authors read, commented and approved the final manuscript.

\section{Funding}

This work was supported by 'Stichting vrienden van Solemio'.

\section{Availability of data and materials}

The datasets used for the current study are available from the corresponding author upon reasonable request.

\section{Declarations}

Ethics approval and consent to participate

Ethics approval was granted by the Leiden University Medical Center research ethics board, and informed consent was obtained from each participant according to the Declaration of Helsinki. All samples and data were coded to maintain the anonymity of the patients.

\section{Consent for publication}

Not applicable.

\section{Competing interests}

HK has performed this work as part of her PhD studies and is currently an employee of Fluidigm. GB is currently an employee of Visualyte.

\section{Author details}

${ }^{1}$ Department of Rheumatology, Leiden University Medical Center, PO box 9600 (Zone C1-R), Albinusdreef 2, 2233, ZA, Leiden, The Netherlands. ${ }^{2}$ Flow Core Facility, Leiden University Medical Center, Leiden, The Netherlands. ${ }^{3}$ Department of Immunology, Leiden University Medical Center, Leiden, The Netherlands. ${ }^{4}$ Institute for Immunity, Transplantation and Infection, Stanford University School of Medicine, Stanford, CA, USA.

Received: 4 February 2021 Accepted: 19 September 2021

Published online: 29 October 2021

\section{References}

1. Fonseka CY, et al. Mixed-effects association of single cells identifies an expanded effector CD4+ T cell subset in rheumatoid arthritis. Sci. Transl. Med. 2018;10. https://pubmed.ncbi.nlm.nih.gov/30333237/.

2. Willemze A, Trouw LA, Toes REM, Huizinga TWJ. The influence of ACPA status and characteristics on the course of RA. Nat. Rev. Rheumatol. 2012;8:144-52.

3. Matthijssen XME, Niemantsverdriet E, Huizinga TWJ, van der Helm-van Mil AHM. Enhanced treatment strategies and distinct disease outcomes among autoantibody-positive and -negative rheumatoid arthritis patients over 25 years: a longitudinal cohort study in the Netherlands. PLoS Med. 2020;17:e1003296.

4. Volkov M, van Schie KA, van der Woude D. Autoantibodies and B Cells: the $A B C$ of rheumatoid arthritis pathophysiology. Immunol. Rev. 2020;294:148-63.

5. Wang Y, et al. Rheumatoid arthritis patients display B-cell dysregulation already in the naïve repertoire consistent with defects in B-cell tolerance. Sci. Rep. 2019;9:19995.

6. Morita T, et al. The proportion of regulatory $T$ cells in patients with rheumatoid arthritis: a meta-analysis. PLoS One. 2016;11:e0162306.

7. Fukui S, et al. M1 and M2 monocytes in rheumatoid arthritis: a contribution of imbalance of M1/M2 monocytes to osteoclastogenesis. Front. Immunol. 2017:8:1958.

8. Paulissen SMJ, et al. CCR6(+) Th cell populations distinguish ACPA positive from ACPA negative rheumatoid arthritis. Arthritis Res. Ther. 2015;17:344.

9. Quandt D, Rothe K, Scholz R, Baerwald CW, Wagner U. Peripheral CD4CD8 double positive T cells with a distinct helper cytokine profile are increased in rheumatoid arthritis. PLoS One. 2014;9:e93293.

10. Pratt AG, et al. A CD4 T cell gene signature for early rheumatoid arthritis implicates interleukin 6-mediated STAT3 signalling, particularly in anti-citrullinated peptide antibody-negative disease. Ann. Rheum. Dis. 2012;71:1374-81.

11. Ornatsky O, Baranov VI, Bandura DR, Tanner SD, Dick J. Multiple cellular antigen detection by ICP-MS. J. Immunol. Methods. 2006;308:68-76.

12. Rao DA, et al. Pathologically expanded peripheral Thelper cell subset drives B cells in rheumatoid arthritis. Nature. 2017;542:110-4. 
13. Zhang F, et al. Defining inflammatory cell states in rheumatoid arthritis joint synovial tissues by integrating single-cell transcriptomics and mass cytometry. Nat. Immunol. 2019;20:928-42.

14. Leite Pereira A, et al. Characterization of phenotypes and functional activities of leukocytes from rheumatoid arthritis patients by mass cytometry. Front. Immunol. 2019;10:2384.

15. de Rooy DPC, van der Linden MPM, Knevel R, Huizinga TWJ, van der Helm-van AHM. Predicting arthritis outcomes-what can be learned from the Leiden Early Arthritis Clinic? Rheumatology. 2011;50:93-100.

16. Arnett FC, et al. The American Rheumatism Association 1987 revised criteria for the classification of rheumatoid arthritis. Arthritis Rheum. 1988;31:315-24

17. van Unen V, et al. Visual analysis of mass cytometry data by hierarchical stochastic neighbour embedding reveals rare cell types. Nat. Commun. 2017;8:1740.

18. Beyrend G, Stam K, Höllt T, Ossendorp F, Arens R. Cytofast: a workflow for visual and quantitative analysis of flow and mass cytometry data to discover immune signatures and correlations. Comput. Struct. Biotechnol. J. 2018;16:435-42.

19. Melsen JE, van Ostaijen-Ten Dam MM, Lankester AC, Schilham MW, van den Akker EB. A comprehensive workflow for applying single-cell clustering and pseudotime analysis to flow cytometry data. J. Immunol. 2020;205:864-71.

20. Akuthota P, Shamri R, Weller PF. Isolation of human eosinophils. Curr. Protoc. Immunol. 2012; Chapter 7:Unit 7.31.

21. Schroeder JT, Bieneman AP. Isolation of human basophils. Curr. Protoc. Immunol. 2016;112:7.24.1-8.

22. Höllt T, et al. Cytosplore: interactive immune cell phenotyping for large single-cell datasets. Comput. Graph. Forum. 2016;35:171-80.
23. Costantini A, et al. Effects of cryopreservation on lymphocyte immunophenotype and function. J. Immunol. Methods. 2003;278:145-55.

24. Lieberman LA, et al. CD62L is not a reliable biomarker for predicting PML risk in natalizumab-treated R-MS patients. Neurology. 2016;86:375-81.

25. Weinberg A, et al. Optimization and limitations of use of cryopreserved peripheral blood mononuclear cells for functional and phenotypic T-cell characterization. Clin. Vaccine Immunol. 2009:16:1176-86.

26. Wang $L$, et al. Standardization of cryopreserved peripheral blood mononuclear cells through a resting process for clinical immunomonitoring-development of an algorithm. Cytometry. A. 2016;89:246-58.

27. Koenigsmann MP, et al. Adhesion molecules on peripheral bloodderived CD34+ cells: effects of cryopreservation and short-term ex vivo incubation with serum and cytokines. Bone Marrow Transplant. 1998:22:1077-85.

28. Aljadi Z, et al. Altered basophil function in patients with chronic kidney disease on hemodialysis. Clin. Nephrol. 2017;88:86-96.

29. Charles N, Hardwick D, Daugas E, Illei GG, Rivera J. Basophils and the T helper 2 environment can promote the development of lupus nephritis. Nat. Med. 2010;16:701-7.

30. Denzel A, et al. Basophils enhance immunological memory responses. Nat. Immunol. 2008;9:733-42.

\section{Publisher's Note}

Springer Nature remains neutral with regard to jurisdictional claims in published maps and institutional affiliations.
Ready to submit your research? Choose BMC and benefit from:

- fast, convenient online submission

- thorough peer review by experienced researchers in your field

- rapid publication on acceptance

- support for research data, including large and complex data types

- gold Open Access which fosters wider collaboration and increased citations

- maximum visibility for your research: over 100M website views per year

At BMC, research is always in progress.

Learn more biomedcentral.com/submissions 